\title{
Trastornos Osteomusculares en Auxiliares de Enfermería en la Unidad de Cuidados Intensivos
}

\author{
MUSCULOSKELETAL DISORDERS IN AUXILIARY NURSING IN INTENSIVE CARE UNIT
}

Álvaro Luis Fajardo Zapata

Profesor Asociado del Departamento de Ingenieria y Ciencias Básicas de la Fundación Universitaria del Área Andina. Bogotá, Colombia.

\begin{abstract}
RESUMEN
Introducción: Los trastornos osteomusculares representan un problema importante en los países en desarrollo por sus altos costos económicos y son reconocidos como una causa importante de ausentismo laboral e incapacidad. Objetivos: Identificar la aparición de trastornos osteomusculares en auxiliares de enfermería que trabajan en la unidad de cuidados intensivos. Materiales y métodos: Estudio descriptivo transversal. Se tomó una muestra 86 auxiliares de enfermería. Se aplicó un cuestionario con 24 preguntas, dentro de un enfoque ergonómico, adaptado a partir del cuestionario Nórdico de Síntomas osteomusculares. Los datos se procesaron en Epi Info 7. Resultados: Se encontró que las auxiliares de enfermería de la unidad de cuidados intensivos presentaron una alta prevalencia de sintomatología osteomuscular (79\%), las partes más afectadas fueron la espalda inferior $(24,5 \%)$, y la espalda superior $(17,5 \%)$. La mayoría de las auxiliares ha recibido incapacidad médica por esta causa (65\%). Conclusiones: Las altas demandas laborales en las unidades de cuidados intensivos generan la aparición de trastornos osteomusculares en las auxiliares de enfermería.
\end{abstract}

(Fajardo A, 2015. Trastornos Osteomusculares en Auxiliares de Enfermería en la Unidad de Cuidados Intensivos. Cienc Trab. May-Ago; 17 [53]: 150-153).

Palabras clave: ENFERMEDADES OCUPACIONALES, LUMBALGIA, PERSONAL DE SALUD, FACTORES DE RIESGO, CUIDADOS INTENSIVOS, TRASTORNOS MUSCULARES.

\section{ABSTRACT}

Introduction: musculoskeletal disorders represent major problems in developing countries because of their high economic costs and are recognized as a major cause of work absenteeism and disability. Objective: identify the onset of skeletal muscle symptoms in nursing assistants working in the intensive care unit. Materials and methods: Descriptive cross-sectional study. A nursing auxiliary 86 sample was taken. A questionnaire with 24 questions, within an ergonomic approach, adapted from the symptoms of Nordic questionnaire musculoskeletal. The data were processed in Epiinfo 3.3.2. Results: found that the auxiliary nurses in the intensive care unit have a high prevalence of symptoms of musculoskeletal (79\%), the most affected part was the lower back (24.5\%), and upper back (17.5\%). Most of the assistants has received medical disability for this cause (65\%). Conclusions: The high labor demands in intensive care units generate the appearance of musculoskeletal disorders in nursing assistants.

Keywords: OCCUPATIONAL DISEASES, LOW BACK PAIN, HEALTH PERSONNEL, RISK FACTORS, INTENSIVE CARE, MUSCULAR DISEASES.

\section{INTRODUCCIÓN}

En el ámbito hospitalario existen diversos factores de riesgo ocupacional capaces de generar trastornos para la salud e incapacidad en los trabajadores. Dentro de los trastornos que generan una mayor morbilidad están los osteomusculares. Dentro del personal expuesto, es el de enfermería quien en razón a las actividades propias de su función y en cumplimiento de las ordenes médicas está sometido a sobreesfuerzos como: bañar al paciente, vestirlo, curarle las heridas, sentarlo o subirlo a la cama o a las

Correspondencia / Correspondence

Alvaro Luis Fajardo

Calle 71 No.13-21

Bogotá, Colombia

Fono: $(57+1) 7449191$

e-mail: afajardo2004@gmail.com, afajardo@areandina.edu.co Recibido: 24 de Mayo de 2015 / Aceptado: 18 de Julio de 2015 sillas, trasladarlo y movilizarlo, transferirlo de una cama a otra, llevarlo al baño; actividades que implican posiciones incomodas, como flexión y rotación de tronco. Todo lo anterior se constituye en un factor de riesgo ergonómico que desencadena la aparición de dolor especialmente en las regiones cervical y lumbar; zonas que están involucradas en la generación de fuerza y movimiento. Adicionalmente hay que agregar que existen otros factores asociados como la frecuencia de la actividad, tiempo, peso del paciente y la dificultad postural requerida para la tarea. ${ }^{1-3}$

Los trastornos osteomusculares representan problemas importantes en los países en desarrollo por sus altos costos económicos y son reconocidos como una causa importante de ausentismo laboral e incapacidad, además del impacto en la productividad y en el bienestar de los trabajadores. La mayoría de las veces son trastornos de carácter crónico lo que dificulta su identificación y la relación con los factores laborales, así como su consideración como enfermedad profesional. $^{4-20}$

Otros factores reconocidos que pueden estar implicados en la aparición de estos trastornos son: la edad, la talla, el índice de masa corporal, el tipo de actividad física, enfermedades concomitantes, el uso de zapatos con tacón alto, tabaquismo, turnos nocturnos, así como factores psicosociales: demandas del empleador, bajo control, 
falta de autonomía, falta de apoyo social, repetitividad, monotonía, insatisfacción laboral, y el número de pacientes que se tiene que atender por turno. ${ }^{12,13,19-23}$

Factores como el ser mujer, tener más de 50 años, una mayor carga física, sobre todo estática, un apoyo social bajo y las cargas domésticas son factores asociados a una mayor incidencia de incapacidad temporal de dolor de espalda. ${ }^{7}$

Se estima que entre un 60 y 90\% de la población sufre dolor lumbar en algún momento de la vida; ocupa el primer lugar en cuanto a frecuencia de trastornos de la salud y es la segunda causa de requerimiento médico en los países industrializados, además se constituye en la primera causa de incapacidad laboral, con la consecuente implicación económica. ${ }^{10,12,18,19,21,22,27-30}$

Diferentes cifras de prevalencia de dolor lumbar en personal de enfermería han sido reportadas dependiendo del país en que se realiza la medición, y van desde el 40\% en la población de enfermeros en general hasta el 81,3\% en enfermeros de unidades de cuidados intensivos. ${ }^{21}$

De acuerdo a Laura Punnett, en el American Journal of Industrial Medicine de 2005, se considera que a nivel mundial, 37 de cada 100 episodios de dolor lumbar son atribuidos al desarrollo de las actividades laborales; y esta fracción varía según se trate de hombres (41\%) o de mujeres (23\%). ${ }^{31}$

En Colombia, las enfermedades osteomusculares ocupan el primer lugar y han ido en aumento a partir de 2006 donde representaron el 82,4\%, en 2007 el 80,2\%, en 2008 el 85\%, en 2009 el 82,7\% y en 2010 el $84 \%$ del total de enfermedades laborales reportadas. ${ }^{32}$

\section{MATERIALES Y MÉTODOS}

Estudio descriptivo transversal realizado en las unidades de cuidado intensivo de dos instituciones hospitalarias de cuarto nivel en Bogotá. La muestra estuvo constituida por 86 auxiliares de enfermería que cumplieron con los criterios de inclusión: auxiliar titulada, laborar exclusivamente en la unidad de cuidados intensivos y querer participar voluntariamente en el estudio. La información se obtuvo mediante un cuestionario con 24 preguntas, donde se identificaron variables sociodemográficas, aspectos propios de la forma de ejecución de las actividades y la aparición de dolor por segmento corporal, así como el tiempo de duración del mismo, dentro de un enfoque ergonómico, adaptado a partir del cuestionario Nórdico de Síntomas Osteomusculares. La base de datos fue elaborada en Excel y los datos se procesaron en Epi Info 7.

\section{RESULTADOS}

El 77\% (66/86) de las auxiliares de enfermería que participaron en éste estudio tenía más de cuatro años de experiencia laboral en la unidad de cuidados intensivos, mientras que el 14\% (12/86) entre uno y dos años, el 9\% (8/86) tenía menos de un año laborando en esta unidad. El 45\% (39/86) manifestó que atendía 4 pacientes por turno, un 23\% (20/86) cinco, el $15 \%$ (13/86) seis y el 17\% (14/86) atendía entre dos y tres pacientes.

La realización de posturas inadecuadas y prolongadas fue reportada como muy a menudo por el 37\% (32/86) de las auxiliares de enfermería, mientras que un 33\% (28/86) reportó que siempre se ve enfrentada a estas posturas, un $29 \%$ (25/86) que a veces las realiza y sólo un 1\% (1/86) manifestó que nunca las realiza. El levantamiento y movilización de cargas es una de las actividades más realizadas ya que el 47\% (40/86) manifestó que siempre las hacía, mientras que un 22\% (19/86) manifestó que las realizaba muy a menudo y el 29\% (25/86) manifestó que a veces las realizaba.

Del total de las auxiliares de enfermería que manifestó sintomatología osteomuscular (68/86), el 63,2\% (43/68), expresó que había sido incapacitado por esta causa, mientras que el 36,8\% (25/68), afirmó no haber recibido incapacidad. Del total que afirmó haber sido incapacitada el 44\% (19/43) le fue dada incapacidad entre uno y tres días, un 37\% (16/43) recibió entre cuatro a siete dias, un 16\% (7/43) entre ocho y 15 días, y un 3\% (1/43) afirmó haber recibido más de 15 días de incapacidad médica. El 18\% (12/68) de las auxiliares que presentaron la sintomatología lo habian reportado a la administradora de riesgo laboral, mientras que un 82\% (56/68) no lo había hecho.

De las auxiliares de enfermería que manifestaron dolor en codos, espalda inferior, caderas, rodillas y tobillos, refirieron que era permanente, mientras que quienes manifestaron sintomatología en cuello, hombros, muñecas y espalda superior refirieron que sólo duraba entre uno y siete días.

De las auxiliares que refirieron sintomatología osteomuscular el 9\% (6/68) manifestó que había sido reubicada laboralmente y un $1 \%(1 / 68)$ refirió haber sido intervenida quirúrgicamente por esta causa.

Este estudio no encontró relación entre el índice de masa corporal y la aparición de trastornos osteomusculares $\left(\mathrm{Chi}^{2}=0.0617\right.$; $\mathrm{p}=0,9696)$, ni con el número de pacientes atendidos por turno (Chi ${ }^{2}$ = 5.46; 0,2429); pero sí se encontró asociación entre la aparición de sintomatología osteomuscular y la edad $\left(\mathrm{Chi}^{2}=35.33 ; \mathrm{p}=0,000\right)$; con el tiempo que la auxiliar llevaba trabajando en la unidad de cuidados intensivos ( $\left.\mathrm{Chi}^{2}=14.20 ; \mathrm{p}=0,0026\right)$, y con el mayor número de hijos de las auxiliares $\left(\mathrm{Chi}^{2}=13.95 ; \mathrm{p}=0,0075\right)$.

\section{DISCUSIÓN}

Este estudio encontró que las auxiliares de enfermería que desarrollan sus actividades laborales en la unidad de cuidado intensivo presentan una alta prevalencia de sintomatología osteomuscular (79\%) producto de las actividades como bañar, movilizar y trasladar pacientes, donde se ven enfrentadas a la realización de posturas inadecuadas y prolongadas, levantamiento y movilización de cargas. Los segmentos corporales que estuvieron implicados con más frecuencia fueron: la espalda inferior $(24,5 \%)$, espalda superior (17,5\%) y las rodillas (13,5\%). El estudio también encontró que la gran mayoría de las auxiliares había recibido incapacidad médica por esta causa (65\%), también encontró que un porcentaje muy bajo de auxiliares había reportado a la administradora de riesgo laboral la sintomatología (18\%), y el 9\% ya había sido reubicada por esta causa.

Lo anterior concuerda con Giovana Pimentel et $\mathrm{al}^{3}$, quien en un estudio que tuvo como objetivo evaluar los síntomas musculoesqueléticos en enfermeras, encontró que de las participantes, el 93\% reportó algún tipo de síntoma musculoesquelético en los últimos 12 meses. La mayor prevalencia de síntomas de acuerdo con las zonas anatómicas fueron: región lumbar (59\%), hombros (40\%), rodillas $(33,3 \%)$ y el cuello $(28,6 \%)$. También encontró que el 29,5\% faltó al trabajo y 47,6\% visitó un médico en los últimos 12 meses debido a los mismos síntomas. 
También Duque et $\mathrm{al}^{21}$, en Colombia, quienes realizaron un estudio para determinar la prevalencia de dolor lumbar en el personal de enfermería, encontraron lumbalgia en el $67,8 \%$ de los participantes, sin encontrar diferencia en cuanto al sexo. Igualmente los resultados concuerdan pero en menor porcentaje con los de Borges et $\mathrm{al}^{22}$, quienes en Venezuela en un estudio sobre lumbalgia ocupacional en personal de enfermería, encontraron una prevalencia del $28 \%$ al momento de realización de la entrevista y del $48 \%$ como percepción de molestia músculoesquelética en el último año. Asímismo los hallazgos de este estudio concuerdan con los de Montoya et $\mathrm{al}^{15}$, quienes reportaron en su estudio una prevalencia del 59.68\% de sintomatología osteomuscular con mayor porcentaje en la región lumbar. También los hallazgos de Vernaza et $\mathrm{al}^{11}$, concuerdan con los de este estudio ya que reportaron un 57\% de sintomatología osteomuscular con predominio del dolor en la región lumbar (56.6\%).

El estudio también encontró que, al parecer, existen factores que pueden estar relacionados con la aparición de la patología osteomuscular como los son: la edad, el tiempo laborado en la unidad de cuidados intensivos, y la mayor carga en actividades domésticas. En cuanto a la edad el estudio, concuerda con el de Capa Verde et $\mathrm{al}^{14} \mathrm{y}$ Gutiérrez et $\mathrm{al}^{25}$, quienes en sus respectivos estudios en trabajadores de diversas empresas encontraron que la sintomatología osteomuscular sobre todo la lumbalgia se hace más evidente en la medida en que los trabajadores tienen más años de edad.

Adicionalmente el estudio concuerda con Martínez et $\mathrm{al}^{27}$, que en su estudio reportaron una incapacidad temporal del 22,5\% en personal hospitalario relacionado con patología Musculoesquelética, mientras que este estudio encontró un 27,3\%.

Es importante recalcar que las auxiliares de enfermería de las unidades de cuidados intensivos realizan una abnegada y ardua labor y la aparición de trastornos osteomusculares se hace evidente en razón a que tienen que movilizar pacientes, quienes dada su incapacidad física por razones obvias, requieren que les realicen el aseo personal, la movilización para evitar las escaras, y otras tantas funciones.

Lo anterior hace que se deba insistir con los programas de capacitación sobre mecánica corporal y además se debe reiterar en la necesidad de que se reporte en la oficina de seguridad y salud en el trabajo la aparición de cualquier sintomatología osteomuscular para tomar las medidas correctivas a fin solucionar el problema que lo generó.

\section{Agradecimientos}

El autor expresa los agradecimientos a las enfermeras: Diana Rodríguez, Carolina Hernández, Martha Copete, Johanna Lemus, Rosa Tamayo, Diana Briceño, de la Facultad de Enfermería de la Fundación Universitaria del Área Andina por sus valiosos aportes a la investigación que dio origen al presente artículo. Adicionalmente expresa sus agradecimientos a Laura Carolina Fajardo Álvarez por la traducción, mejoramiento y síntesis del mismo.

\section{Fuentes de financiación}

La presente investigación fue parcialmente financiada por la Fundación Universitaria del Área Andina. Los demás gastos fueron asumidos por los investigadores. 
1. Colombia. Ministerio de la Protección Social. Guia de Atención Integral Basada en la Evidencia para Desórdenes Músculo-Esqueléticos (DME) relacionados con Movimientos Repetitivos de Miembros Superiores (Sindrome de Túnel Carpiano, Epicondilitis y Enfermedad de De Quervain (GATI-DME) [en línea]. Bogotá: Ministerio de la Protección Social; 2006. [citado 25 ago 2014]. Disponible en: http://www.susalud.com/guias/guias_mmss.pdf

2. Estevam M, Costa A. Avaliação de uma cadeira de banho utilizada em ambiente hospitalar: uma abordagem ergonómica. Rev Bras Enferm. 2005; 58(4):405-410.

3. Pimentel $G$, Costa $A$, Rodriguez F. La prevalencia de sintomas osteomusculares en enfermeria. Rev Latino-Am Enfermagem. 2003;11(5): 608-613.

4. Morata $M$, Ferrer V. Interacción entre estrés ocupacional, estrés psicológico y dolor lumbar: un estudio en profesionales sanitarios de traumatología y cuidados intensivos. Mapfre Medicina. 2004;15(3):199-211

5. Kee D, Seo RS. Musculoskeletal disorders among nursing personnel in Korea. Int J Ind Ergonom. 2007;37:207-212.

6. Da Costa B, Ramos E. Risk Factors for Work-Related Musculoskeletal Disorders: A Systematic Review of Recent Longitudinal Studies. Am J Ind Med. 2010; 53:285-323.

7. Burillo T, Mayordomo J, Fernández C, Escriba V. Absentismo laboral por dolor de espalda en personal hospitalario: estudio de cohortes. Fundación Mapfre. 2006; 17(1):3-13.

8. Piedrahita L. Evidencias epidemiológicas entre factores de riesgo en el trabajo y los desórdenes músculo-esqueléticos. Mapfre Medicina. 2004;15(3):212-221.

9. Díez $M$, Garasa $A$, Goretti $M$, Eranzus J. Trastornos músculo-esqueléticos de origen laboral. Instituto Navarro de Salud Laboral [en línea]. Pamplona: Instituto Navarro de Salud Laboral; 2007 [citado 20 jun 2014]. Disponible en: https://www.navarra.es/NR/rdonlyres/76DF548D-769E-4DBF-A18E-8419F3A9 A5FB/145886/TrastornosME.pdf

10. Ruiz $A$, González $R$, Sierra M. Patología osteomuscular en centros sanitarios de la provincia de Málaga. Enfermeria docente. 2005;(81):26-33.

11. Vernaza P, Sierra C. Dolor músculo-esquelético y su asociación con factores de riesgo ergonómicos, en trabajadores administrativos. Rev salud pública. 2005; 7(3):317-326 .

12. Bernard B. A critical review of epidemiologic evidence for work-related musculoskeletal disorders of the neck, upper extremity and low back [on line]. In: National Institute for Occupational Safety and Health. Musculoskeletal disorders and workplace factors. Cincinnati: NIOSH; 1997 [citado18 Jun 2014]. (NIOSH Publication, 97-141). Disponible en: http://www.cdc.gov/niosh/docs/ 97-141/

13. Robaina C, León I, Sevilla D. Epidemiología de los trastornos osteomioarticulares en el ambiente laboral. Rev Cubana Med Gen Integr. 2000; 16(6):531-39

14. Capa Verde M, Cezar M, De Souza J, Santos M. Prevalencia de enfermedades musculoesqueléticas entre trabajadores portuarios apulsos. Rev Latino-Am Enfermagem [en línea] 2012;20(2) [citado18 jul 2014]. Disponible en: http:// www.scielo.br/pdf/rlae/v20n2/es_05.pdf

15. Montoya M, Palucci M, Do carmo M, Taubert F. Lesiones osteomusculares en trabajadores de un hospital mexicano y la ocurrencia del ausentismo. Cienc Enferm. 2010;(2):35-46.

16. Castro E, Múnera J, Sanmartín M, Valencia N, Valencia N, González E. Efectos de un programa de pausas activas sobre la percepción de desórdenes músculo- esqueléticos en trabajadores de la Universidad de Antioquia. Revista Educ Fis Deport. 2011;30(1):389-399.

17. Viaño J, Díaz $P$, Martínez $A$. Trastornos músculo-esqueléticos en músicos instrumentalistas estudiantes de secundaria y universitarios. Revista de Investigación en Educación. 2010;8:83-96

18. Bellorin $M$, Sirit $Y$, Rincón $C$, Amórtegui $M$. Sintomas Músculo Esqueléticos en trabajadores de una empresa de construcción Civil. Salud de los Trabajadores. 2007;15(2):89-98

19. Bohuslav Málek. Riesgos para la salud en obras subterráneas. En: OIT. Enciclopedia de la Salud y Seguridad en el Trabajo [en línea]. Ginebra: OIT; 2007. [citado 10 mar 2014]. Disponible en: http://www.cdc.gov/elcosh/docs/\% 20d0200/d000279/d000279s.pdf

20. Zhuang A, Stobbe T, Hsiao T, Collins J, Hobbs G. Biomechanical evaluation of assistive devices for transferring residents. Appl Ergon. 1999;30:285-294.

21. Duque I, Zuluaga D, Pinilla A. Prevalencia de lumbalgia y factores de riesgo en enfermeros y auxiliares de la ciudad de Manizales. Hacia Promoc Salud. 2011; 16(1):27-38.

22. Borges $A$, Maizlish $N$, Loreto V. Lumbalgia ocupacional en enfermeras venezolanas. Salud de los trabajadores. 2004;12:19-32.

23. Salazar C, Ricardo J, Ararat J, Castillo C, Ríos C. Factores de riesgo ergonómico relacionados a sintomatología de dolor musculoesquelético en descortezadores de la cooperativa agroforestal del Cauca (COOTRAFORC). Rev Cubana Salud Trabajo. 2011;12(1):28-38.

24. Bongers $P$, De Winter $C$, Kompier M, Hildebrandt V. Psychosocial factors at work and musculoskeletal disease. Scand J Work Env Hea. 1993;19: 297-312.

25. Gutiérrez $A$, Del Barrio $A$, Ruiz C. Factores de riesgo y patología lumbar ocupacional. Mapfre Medicina. 2001;12(3):204-213

26. Eriksen W, Bruusgaard D, Knardahl S. Work factors as predictors of intense or disabling low back pain; a prospective study of nurses' aides. Occup Environ Med. 2004;61:398-404

27. Martínez $M$, Vázquez M. Estudio epidemiológico del absentismo laboral en el personal hospitalario por dolor de espalda. Rehabilitación. 2002;36 (3):137-142.

28. Valecillo M, Quevedo A, Lubo A, Dos Santos Alberto, Montiel M, Camejo M., Sánchez M. Sintomas musculoesqueléticos y estrés laboral en el personal de enfermeria de un hospital militar. Salud de los trabajadores. 2009;17(2):85-95.

29. Ocaña U. Lumbalgia ocupacional y discapacidad laboral. Rev Fisioter. 2007; 6(2):17-26

30. Colombia. Ministerio de la Protección Social. Guía de Atención Integral Basada en la Evidencia para Dolor Lumbar Inespecifico y Enfermedad Discal Relacionados con la Manipulación Manual de Cargas y otros Factores de Riesgo en el Lugar de Trabajo (GATI- DLI- ED) [en línea]. Bogotá: Ministerio de la Protección Social; 2006. [citado12 Abr 2014]. Disponible en: http:// www.susalud.com/guias/dolor_lumbar.pdf

31. Punnett $L$, Pruss A, Phillips S. Estimating the global burden of low back pain attributable to combined occupational exposures. Am J Ind Med. 2005;48 (6):459.

32. Colombia. Federación de Aseguradores Colombianos (FASECOLDA). Estadisticas sistema general de riesgos profesionales año 2010 [en línea]. Bogotá: FASECOLDA; 2015 [citado 15 may 2014]. Disponible en: http://www.fasecolda. com/index.php/ramos/riesgos-laborales/estadisticas-del-ramo/ 